\title{
BMJ Open Whole blood versus component therapy for haemostatic resuscitation of major bleeding: a protocol for a systematic review and meta-analysis
}

\author{
Anna E Ssentongo (D) , , 2 Paddy Ssentongo (1) , ${ }^{1,3}$ Emily Heilbrunn (D) ,1 \\ Lacee Laufenberg Puopolo, ${ }^{2}$ Vernon M Chinchilli, ${ }^{1}$ John Oh, ${ }^{2}$ Joshua Hazelton ${ }^{2}$
}

To cite: Ssentongo AE, Ssentongo P, Heilbrunn E, et al. Whole blood versus component therapy for haemostatic resuscitation of major bleeding: a protocol for a systematic review and meta-analysis. BMJ Open 2021;11:e043967. doi:10.1136/ bmjopen-2020-043967

- Prepublication history and additional supplemental material for this paper are available online. To view these files, please visit the journal online (http://dx.doi.org/10.1136/ bmjopen-2020-043967).

AES and PS contributed equally.

Received 18 August 2020 Accepted 28 July 2021

Check for updates

(C) Author(s) (or their employer(s)) 2021. Re-use permitted under CC BY-NC. No commercial re-use. See rights and permissions. Published by BMJ.

${ }^{1}$ Department of Public Health Sciences, Penn State College of Medicine, Hershey, Pennsylvania, USA

${ }^{2}$ Department of Trauma Surgery, Penn State College of Medicine, Hershey, Pennsylvania, USA ${ }^{3}$ Department of Engineering, Science, and Mechanics, Penn State, University Park, Pennsylvania, USA

Correspondence to Anna E Ssentongo; assentongo@pennstatehealth. psu.edu

\section{ABSTRACT}

Introduction There is a renewed interest in the use of whole blood (WB) to manage patients with lifethreatening bleeding. We aimed to estimate mortality and complications risk between WB and blood component therapy for haemostatic resuscitation of major bleeding. Methods We will conduct a systematic review and meta-analysis of studies published between 1 January 1980 and 1 January 2020, identified from PubMed and Scopus databases. Population will be patients who require blood transfusion (traumatic operative, obstetric and gastrointestinal bleeding). Intervention is WB transfusion such as fresh WB (WB unit stored for less than 48 hours), leukoreduced modified WB (with platelets removed during filtration), warm fresh WB (stored warm at $22^{\circ} \mathrm{C}$ for up to 8 hours and then for a maximum of an additional 24 hours at $4^{\circ} \mathrm{C}$ ). The primary outcomes will be the 24-hour and 30-day survival rates (in-hospital mortality). Comparator is blood component therapy (red blood cells, fresh-frozen plasma and platelets given together in a 1:1:1 unit ratio). The Cochrane risk of bias tool for randomised controlled trials and Risk Of Bias In Non-randomised Studies - of Interventions (ROBINS-I) for observation studies will be used to assess the risk of bias of included studies. We will use random-effects models for the pooling of studies. Interstudy heterogeneity will be assessed by the Cochran $Q$ statistic, where $p<0.10$ will be considered statistically significant and quantified by $\mathrm{I}^{2}$ statistic, where $\mathrm{I}^{2} \geq 50 \%$ will indicate substantial heterogeneity. We will perform subgroup and meta-regression analyses to assess geographical differences and other study-level factors explaining variations in the reported mortality risk. Results will be reported as risk ratios and their $95 \%$ Cls.

Ethics and dissemination No ethics clearance is required as no primary data will be collected. The results will be presented at scientific conferences and published in a peer-reviewed journal.

\section{INTRODUCTION}

Over the past three decades, there is a renewed interest in using whole blood (WB) to manage patients with life-threatening bleeding. Robertson initially established the foundation for blood transfusions for traumatic injuries during the First World War
Strengths and limitations of this study

- The systematic review and meta-analysis will update the available evidence on mortality and morbidity risk associated with whole blood use compared with component therapy in treating life-threatening bleeding.

- We adhered to Preferred Reporting Items for Systematic Reviews and Meta-Analyses Protocols guidelines to ensure transparency of the study.

- Between-study heterogeneity is a limitation. We will use random-effects meta-regression models to characterise the sources of heterogeneity.

when blood banking during military combat became available. ${ }^{1}$ However, civilian establishment of organised blood banking lagged by several decades and became readily accessible during the Second World War. Stored WB was the mainstay of transfusion through the beginning of the Vietnam era. In 1965, during the Vietnam War, blood component therapy was introduced, and by the 1970s, WB resuscitation had nearly ceased. ${ }^{2}$ Component therapy, including packed red blood cells (PRBC), fresh frozen plasma (FFP) and platelets (PLT), has been adopted as the gold standard for both military and civilian trauma resuscitation.

Strategies in component resuscitation have evolved over the past several decades, with the adoption of damage control resuscitation (DCR). DCR principles include early administration of blood products in a balanced ratio, prevention and correction of coagulopathy, and minimisation of crystalloid fluid resuscitation. ${ }^{3}$ Typical initial resuscitation using a massive transfusion protocol uses component therapy from universally compatible donors before laboratory testing. The concept of a balanced component resuscitation that supports achieving haemostasis with 
transfusion of PRBC, FFP and PLT that approximates WB has been largely supported by recent evidence. ${ }^{45}$ While component therapy has dominated civilian resuscitation of haemorrhagic shock, fresh $\mathrm{WB}$ (stored at $22^{\circ} \mathrm{C}$ for 24 hours) has continued to be used in military trauma resuscitation in austere environments where the storage of component therapy is unavailable. In fact, over 6000 units of type-specific warm fresh WB were transfused during the Iraq and Afghanistan conflicts to patients with severe haemorrhage, and these patients showed increased 24-hour and 30-day survival and decreased transfusion requirements. ${ }^{6} 7$ However, warm fresh WB still only comprised $4 \%$ of transfusions during this era, reflecting the current practice of using component therapy when available. ${ }^{6}$

Civilian interest in the use of WB for resuscitation of traumatic haemorrhagic shock has resurged in the past decade. Unlike in austere military environments, civilian usage of WB has been in the form of cold-stored WB, stored for up to 21 days between $1^{\circ} \mathrm{C}$ and $6^{\circ} \mathrm{C}$. Stored WB has an established safety profile; over 350000 units were transfused during the Vietnam war with low rates of haemolysis. ${ }^{8}$ Low-titre, leucocyte-reduced, PLT-sparing group $\mathrm{O} W B$ has been used in several small series at three level 1 trauma centres in the USA with initial published data suggesting a trend towards decreased transfusion requirements, but studies have been small and underpowered to detect a survival benefit. ${ }^{910}$ However, these studies did establish a safety profile for the practice of transfusing group $\mathrm{OWB}$, with no reports of transfusion reactions or differences in serum haptoglobin as a marker of haemolysis. ${ }^{1011}$ The risk of haemolysis caused by the transfusion of group $\mathrm{O}$ low-titre $\mathrm{WB}$ to a non-group $\mathrm{O}$ recipient is low. 812

There has been preliminary investigation by one series into coagulation markers as assessed by thromboelastography, with improvement in markers of coagulopathy seen in groups receiving WB and PLT transfusion. ${ }^{13}$ However, there still remains a paucity of literature assessing the effects of WB in the resuscitation of haemorrhagic shock on mortality, total transfusion requirements and need for damage control. Systematic reviews assessing the effect of WB transfusion versus component therapy on outcomes have limitations, including selection bias, age groups studied, limited outcomes assessed and did not address sources of heterogeneity in results using randomeffects meta-regression and subgroup analyses. ${ }^{14-16}$ In this study, we seek to fill this gap by evaluating mortality rates and identifying potential complications of WB transfusion, including haemolysis and thrombotic events. We propose to assess sources of heterogeneity (which are very common in meta-analysis) through random-effects metaregression and subgroup analyses.

\section{Objectives}

This study aims to present a protocol for systematic review and meta-analysis to compare 24-hour and 30-day survival in WB use and component therapy for haemostatic resuscitation of major bleeding.

\section{Specific aims}

- Compare differences between 24-hour and 30-day survival in groups of patients receiving WB or blood components (pRBC, PLT and FFP).

- To estimate morbidity such as acute kidney injury, sepsis, venous thromboembolism, acute respiratory distress syndrome, 24 hours transfusion volume, coagulation abnormality, intensive care unit (ICU) length of stay and ventilation days in a group receiving $\mathrm{WB}$ compared with component therapy.

\section{Review question}

What is the 24-hor and 30-day survival rates following the use of $\mathrm{WB}$ or blood component therapy?

\section{METHODS}

This protocol was reported according to the guidelines of the Preferred Reporting Items for Systematic Reviews and Meta-Analyses Protocols. ${ }^{17} 18$ See online supplemental table 1 .

\section{Patient and public involvement}

Patients or the public were not involved in the design, or conduct, or reporting, or dissemination plans of our research.

\section{Eligibility/ inclusion criteria}

Studies will be selected according to the PICO criteria: Patient (P), Intervention (I), Comparator $(\mathrm{C})$, and Outcome(s) of interest $(\mathrm{O})$. We will exclude patients who received WB outside the above clinical settings.

\section{Patients}

Adult and paediatric patients who require blood transfusion (symptomatic anaemia with large-volume deficits, traumatic operative, obstetric and gastrointestinal bleeding). Patients will be eligible regardless of age, sex or geographical location. Randomised controlled trials (RCTs) and observational studies will be included in this meta-analysis. Studies published between 1 January 1980 and 1 January 2020 will be screened, and no language limitation will be imposed. We will exclude studies not conducted in humans and meeting abstracts, review papers and commentaries.

\section{Intervention}

WB transfusion such as fresh WB (WB unit stored for less than 48 hours), leucoreduced modified WB (with PLT removed during filtration), warm fresh WB (stored warm at $22^{\circ} \mathrm{C}$ for up to 8 hours and then for a maximum of an additional 24 hours at $\left.4^{\circ} \mathrm{C}\right)$. 


\section{Outcome (S) of interest}

The primary outcomes will be the 24-hour and 30-day survival rates (in-hospital mortality). Secondary outcomes will include acute kidney injury, sepsis, venous/arterial thromboembolism, acute respiratory distress syndrome, 24 hours transfusion volume, coagulation abnormality, haemolysis, ICU length of stay and ventilation days.

\section{Comparator}

Blood component therapy ((RBCs, fresh-FFP, andPLTs given together in a 1:1:1 unit ratio).

\section{Database searches}

The following databases will be searched: PubMed (MEDLINE), Scopus, OVID (HEALTH STAR), OVID (MEDLINE) and Joanna Briggs Institute EBP databases. We will use a snowballing method to search the citation lists of included papers using the 'cited by' tool in Google Scholar. We will contact corresponding authors of published or ongoing studies for information regarding missing data.

\begin{tabular}{l}
\hline Search terms \\
Our keyword search will be based on Medical Subject \\
Headings (MeSH) with various combinations of the main \\
search: "Whole blood" OR "Component Therapy" AND \\
"transfusion." We will tailor the search terms with each \\
database using Boolean operators, truncations, proximity \\
operators, and Medical Subject Headings. For a complete \\
list of search terms used for MEDLINE database, see online \\
supplemental table 2 .
\end{tabular}

\section{Title, Abstract and full-text screening}

The citations will be downloaded into the Endnote software, and we will exclude duplicate articles. Two reviewers (AES and EH) will independently screen studies in two stages. In the first stage, the reviewers will independently screen titles and abstracts and document reasons for exclusion if applicable. In the second stage, full-text versions of selected abstracts will be downloaded/retrieved and assessed independently by the two reviewers (AES and $\mathrm{EH})$.

\section{Data extraction}

Data will be extracted from eligible papers. Disagreements will be discussed with a third reviewer (PS) to reach a consensus. If a publication is not available in English, reviewers will seek translation. We will extract the following information: first author, country in which the study was conducted, year of publication, study period, hospital specialty, whether resuscitation was indicated, research methodology, total sample size, survival rates, complications rates, percent of study sample that was male, mean age at transfusion, risk ratios (RR) of mortality and secondary outcomes. In case of missing data, one attempt will be made to contact the corresponding author of the associated study by email.
Assessment of methodological quality of the papers

Two authors (AES and $\mathrm{EH}$ ) will independently assess the quality of the papers included in the review. The Cochrane risk of bias tool for randomised control trials (RCT) and Risk Of Bias In Non-randomised Studies - of Interventions (ROBINS-I) for observation studies. Metaregression analysis will be conducted to assess the effect of the study quality on the primary outcome.

\section{Data synthesis and analysis}

The primary outcomes will be mortality risk associated with WB and component therapy. We will use randomeffects models for the pooling of studies. ${ }^{19}$ We will separate civilian from military patients during the analysis and reporting of the result. Interstudy heterogeneity will be assessed by the Cochran $Q$ statistic, where $p<0.10$ will be considered statistically significant and quantified by $\mathrm{I}^{2}$ statistic, where $\mathrm{I}^{2} \geq 50 \%$ will indicate substantial heterogeneity. We will perform subgroup and meta-regression analyses to assess geographical differences and other studylevel factors that could explain variations in the reported mortality risk. These will include median/mean age, race and sex proportions, civilian versus military patients and the compositions of component therapy. Results will be reported as RR and their 95\% CIs. Publication bias will be assessed by visual inspection of funnel plots and the Egger and Begg tests when $\geq 10$ study comparisons are available. In the presence of publication bias, adjustment for funnel plot asymmetry will be done by imputing missing study data using the Duval and Tweedie trim-and-fill method.

\section{Additional analyses}

Several blood components will be documented in the table format. If too much heterogeneity exists in the component therapy, we will not pool the results in a metaanalysis. Nevertheless, supposing enough publications are included, various blood components and RBC, PLT and plasma ratios will be used as regressors or groups in meta-regression and subgroup analysis, respectively.

\section{Ethics and dissemination}

No ethics clearance is required as no primary data will be collected. The results of this systematic review and metaanalysis will be presented at scientific conferences and published in a peer-review journal.

\section{Presentation of results and reporting}

The results of the final manuscript will be reported according to the guidelines of the Preferred Reporting Items for Systematic Reviews and Meta-Analyses.

\section{Potential amendments}

The protocol was written in 2020, and the study is expected to be completed by 2021 . We do not foresee the need for amendments to this protocol, but they will be registered and reported if they arise.

\section{CONCLUSION}

In this systematic review and meta-analysis, we will synthesise the current literature on WB use versus PRBC, PLT 
and FFP component therapy to manage life-threatening haemorrhage. This meta-analysis will include RCT and observational studies to assess the association of blood transfusion types with mortality and morbidity. This study will inform haemostatic resuscitation of major bleeding when assessing which blood therapy optimises survival and reduce morbidity.

Contributors AES, JH and J0 conceived this study. AES, PS, EH and JH drafted the protocol. JO, PS, LLP, JH, VMC, AES and EH critically reviewed the protocol and provided comments. All authors approved the final protocol.

Funding The authors have not declared a specific grant for this research from any funding agency in the public, commercial or not-for-profit sectors.

Competing interests None declared.

Patient consent for publication Not applicable.

Provenance and peer review Not commissioned; externally peer reviewed.

Supplemental material This content has been supplied by the author(s). It has not been vetted by BMJ Publishing Group Limited (BMJ) and may not have been peer-reviewed. Any opinions or recommendations discussed are solely those of the author(s) and are not endorsed by BMJ. BMJ disclaims all liability and responsibility arising from any reliance placed on the content. Where the content includes any translated material, BMJ does not warrant the accuracy and reliability of the translations (including but not limited to local regulations, clinical guidelines, terminology, drug names and drug dosages), and is not responsible for any error and/or omissions arising from translation and adaptation or otherwise.

Open access This is an open access article distributed in accordance with the Creative Commons Attribution Non Commercial (CC BY-NC 4.0) license, which permits others to distribute, remix, adapt, build upon this work non-commercially, and license their derivative works on different terms, provided the original work is properly cited, appropriate credit is given, any changes made indicated, and the use is non-commercial. See: http://creativecommons.org/licenses/by-nc/4.0/.

\section{ORCID iDs}

Anna E Ssentongo http://orcid.org/0000-0001-9104-1323

Paddy Ssentongo http://orcid.org/0000-0003-1565-5731

Emily Heilbrunn http://orcid.org/0000-0002-9868-5495

\section{REFERENCES}

1 Robertson $\mathrm{OH}$. Transfusion with preserved red blood cells. Br Med J 1918;1:691-5.

2 Bahr MP, Yazer MH, Triulzi DJ, et al. Whole blood for the acutely haemorrhaging civilian trauma patient: a novel idea or rediscovery? Transfus Med 2016;26:406-14.

3 Holcomb JB, Tilley BC, Baraniuk S, et al. Transfusion of plasma, platelets, and red blood cells in a 1:1:1 vs a 1:1:2 ratio and mortality in patients with severe trauma: the PROPPR randomized clinical trial. JAMA 2015;313:471-82.

4 Holcomb JB, del Junco DJ, Fox EE, et al. The prospective, observational, multicenter, major trauma transfusion (PROMMTT) study: comparative effectiveness of a time-varying treatment with competing risks. JAMA Surg 2013;148:127-36.

5 Del Junco DJ, Holcomb JB, Fox EE. Resuscitate early with plasma and platelets or balance blood products gradually: findings from the prospective, observational, multicenter, major trauma transfusion (PROMMTT) study. J Trauma Acute Care Surg 2013;75:S24.

6 Spinella PC. Warm fresh whole blood transfusion for severe hemorrhage: U.S. military and potential civilian applications. Crit Care Med 2008;36:S340-5.

7 Spinella PC, Perkins JG, Grathwohl KW, et al. Warm fresh whole blood is independently associated with improved survival for patients with combat-related traumatic injuries. J Trauma 2009;66:S69-76.

8 Berséus O, Boman K, Nessen SC, et al. Risks of hemolysis due to anti-A and anti-B caused by the transfusion of blood or blood components containing ABO-incompatible plasma. Transfusion 2013;53 Suppl 1:114S-23.

9 Cotton BA, Podbielski J, Camp E, et al. A randomized controlled pilot trial of modified whole blood versus component therapy in severely injured patients requiring large volume transfusions. Ann Surg 2013;258:527-33.

10 Yazer MH, Jackson B, Sperry JL, et al. Initial safety and feasibility of cold-stored uncrossmatched whole blood transfusion in civilian trauma patients. J Trauma Acute Care Surg 2016;81:21-6.

11 Tsai T-H, Lieu A-S, Hwang S-L, et al. A comparative study of the patients with bilateral or unilateral chronic subdural hematoma: precipitating factors and postoperative outcomes. J Trauma 2010;68:571-5

12 Spinella PC, Pidcoke HF, Strandenes G, et al. Whole blood for hemostatic resuscitation of major bleeding. Transfusion 2016;56 Suppl 2:S190-202.

13 Rahbar E, Cardenas JC, Matijevic N, et al. Trauma, time, and transfusions: a longitudinal analysis of coagulation markers in severely injured trauma patients receiving modified whole blood or component blood products. Shock 2015;44:417-25.

14 Avery P, Morton S, Tucker $\mathrm{H}$, et al. Whole blood transfusion versus component therapy in adult trauma patients with acute major haemorrhage. Emerg Med J 2020;37:370-8.

15 Cruciani M, Franchini M, Mengoli C, et al. The use of whole blood in traumatic bleeding: a systematic review. Intern Emerg Med 2021;16:209-20.

16 Crowe E, DeSantis SM, Bonnette A, et al. Whole blood transfusion versus component therapy in trauma resuscitation: a systematic review and meta-analysis. J Am Coll Emerg Physicians Open 2020;1:633-41.

17 Moher D, Shamseer L, Clarke M, et al. Preferred reporting items for systematic review and meta-analysis protocols (PRISMA-P) 2015 statement. Syst Rev 2015;4:1.

18 Shamseer L, Moher D, Clarke M, et al. Preferred reporting items for systematic review and meta-analysis protocols (PRISMA-P) 2015: elaboration and explanation. BMJ 2015;350:g7647.

19 Balduzzi S, Rücker G, Schwarzer G. How to perform a meta-analysis with R: a practical tutorial. Evid Based Ment Health 2019;22:153-60. 